\title{
Diagnostic value and prognostic evaluation of dynamic video- electroencephalogram monitoring in children with epilepsy
}

\author{
XIAOBO WU, YING SU, WEI LIU, XIAOYU JIA, YANLING ZHANG, XINYANG ZHANG and GUILAN WANG
}

Department of Pediatrics, The First Hospital of Qinhuangdao, Qinhuangdao, Hebei 066000, P.R. China

Received May 5, 2017; Accepted July 14, 2017

DOI: $10.3892 / \mathrm{etm} .2017 .4822$

\begin{abstract}
The diagnostic and prognostic value of dynamic video-electroencephalogram (V-EEG) monitoring in children with epilepsy were investigated. From February 2014 to February 2016, in total 200 children with epilepsy were selected during their stay in the First Hospital of Qinghuangdao and were randomly allocated to a dynamic and a conventional V-EEG monitoring group $(n=100)$. The detection rate of epileptiform discharges in the two groups was evaluated. The V-EEG monitoring index was used to select treatment methods for patients with epilepsy. After 3 months, the patients were rechecked. Disease features and incidence of epilepsy were recorded. There were no significant differences in the course of disease, seizure frequency and age between the two groups $(\mathrm{P}>0.05)$. The detection rate of epileptiform discharges in the dynamic V-EEG was significantly higher than in the routine monitoring group $(\mathrm{P}<0.01)$. The accuracy and specificity of monitoring in the V-EEG were significantly higher than in the routine monitoring group $(\mathrm{P}<0.01)$. Seizure frequency and number of epilepsy attacks in patients in the V-EEG group were significantly lower than in the routine monitoring group $(\mathrm{P}<0.01)$. Dynamic V-EEG can improve epilepsy detection rate. The high accuracy and specificity of dynamic V-EEG suggest that it may be of great clinical value in the diagnosis and prognosis of epilepsy.
\end{abstract}

\section{Introduction}

One of the common diseases of the nervous system, epilepsy is a paroxysmal disease that is mainly caused by the abnormal discharge of brain neurons or synchronization activities (1-3). Epilepsy can occur in various forms and onset is often uncertain, though it occurs mainly in children. These factors may complicate clinical diagnosis and abnormal neuronal discharge

Correspondence to: Ms. Xiaobo Wu, Department of Pediatrics, The First Hospital of Qinhuangdao, 258 Wenhua North, Qinhuangdao, Hebei 066000, P.R. China

E-mail: ulogdm@163.com

Key words: dynamic video-electroencephalogram, prognostic evaluation, epilepsy recorded by electroencephalogram (EEG) is often used in the diagnosis (4-6). Dynamic EEG is a commonly used tool for the detection of epilepsy, but it has the disadvantages of high false-positive rate and poor synchronization accuracy $(7,8)$. Dynamic EEG combined with video observation can be used for the long-term simultaneous monitoring of the performance of patients with epilepsy and can be used to diagnose the onset of epilepsy $(9,10)$. The aim of this study is to investigate the differences between dynamic V-EEG and conventional EEG in the diagnosis of epilepsy and identify which method has better accuracy, efficiency and specificity for diagnosis and prognosis.

\section{Materials and methods}

Subjects. Patients involved in this study were all selected from February 2014 to 2016 while receiving neurological treatment in the First Hospital of Qinghuangdao. Included patients were all found to have different degrees of epilepsy-like symptoms by clinical examination and were all subsequently diagnosed with epilepsy. Consumptive diseases and other diseases that can cause EEG changes were ruled out in all included patients. Legal guardians of the included children signed the informed consent form and detailed clinical data were recorded for all patients.

Grouping. The 200 patients were randomly allocated to either the dynamic V-EEG or the conventional monitoring group. The age, sex, weight, number of seizures and course of disease, as well as other clinical and pathological data were subjected to statistical analysis. According to their assigned monitoring group, patients were subjected to $24 \mathrm{~h}$ of dynamic V-EEG or conventional EEG monitoring.

Methods. The electrodes of the dynamic V-EEG recorder (Vidy dynamic video EEG recording box; Biologics Co., London, UK) were set according to the international 10/20 system and reference electrodes were placed at the ear position on both sides. The electrodes were fixed in a double-layer elastic cap. Patients were subjected to $24 \mathrm{~h}$ of dynamic EEG monitoring and clinical manifestations and EEG changes were recorded. The experiment was induced by blinking and hyperventilation. Epilepsy symptoms were recorded as clinical manifestations and epilepsy-like EEG for $72 \mathrm{~h}$ (if during $24 \mathrm{~h}$ of monitoring, a patient exhibited epilepsy-like EEG, 
Table I. General information and clinicopathological features of patients.

\begin{tabular}{|c|c|c|c|c|c|c|}
\hline \multirow[b]{2}{*}{ Groups } & \multirow[b]{2}{*}{ Patients } & \multirow[b]{2}{*}{ Mean age (years) } & \multicolumn{2}{|c|}{ Sex } & \multirow{2}{*}{$\begin{array}{l}\text { Mean duration } \\
\text { of disease (years) }\end{array}$} & \multirow{2}{*}{$\begin{array}{l}\text { Seizure frequency } \\
\quad \text { (per week) }\end{array}$} \\
\hline & & & Male (n) & Female (n) & & \\
\hline Dynamic V-EEG & 100 & $8.53 \pm 3.86$ & 54 & 46 & $3.27 \pm 2.52$ & $2.18 \pm 0.86$ \\
\hline Conventional & 100 & $8.09 \pm 3.27$ & 55 & 45 & $3.47 \pm 2.18$ & $2.32 \pm 0.78$ \\
\hline$\chi^{2}$ & & & & & 0.03 & 0.08 \\
\hline P-value & & $>0.05$ & $>0.05$ & & $>0.05$ & $>0.05$ \\
\hline
\end{tabular}

V-EEG, video-electroencephalogram.

Table II. Classification of epilepsy types in the two groups.

\begin{tabular}{lcccccccc}
\hline Groups & $\begin{array}{c}\text { Partial } \\
\text { seizures (n) }\end{array}$ & $\begin{array}{c}\text { Spastic } \\
\text { seizures (n) }\end{array}$ & $\begin{array}{c}\text { Typical } \\
\text { absence } \\
\text { seizures (n) }\end{array}$ & $\begin{array}{c}\text { Atypical } \\
\text { absence } \\
\text { seizures (n) }\end{array}$ & $\begin{array}{c}\text { Tonic-clonic } \\
\text { seizures (n) }\end{array}$ & $\begin{array}{c}\text { Atonic } \\
\text { seizures (n) }\end{array}$ & $\begin{array}{c}\text { Myoclonic } \\
\text { seizures (n) }\end{array}$ & $\begin{array}{c}\text { Complex } \\
\text { seizures (n) }\end{array}$ \\
\hline Dynamic V-EEG & 58 & 15 & 3 & 1 & 5 & 8 & 1 & 9 \\
Conventional & 55 & 16 & 4 & 2 & 4 & 10 & 2 & 7 \\
P-value & $>0.05$ & $>0.05$ & $>0.05$ & $>0.05$ & $>0.05$ & $>0.05$ & $>0.05$ & $>0.05$ \\
\hline
\end{tabular}

V-EEG, video-electroencephalogram.

monitoring was continued for $72 \mathrm{~h}$ ). EEGs in the routine monitoring group were recorded using a dynamic EEG monitoring system (Weisi Technology Co., Jiangsu, China); all other steps were the same as those of the dynamic V-EEG monitoring group.

Intervention treatment. Epilepsy seizure types detected by both dynamic V-EEG and conventional EEG monitoring were classified according to the criteria established by the International League Against Epilepsy (ILAE). Patients were given appropriate drugs for routine treatment. Patients in the dynamic V-EEG and routine monitoring group were subjected to the same drug treatment. After 3 months, the patients were rechecked. Disease features and incidence of epilepsy attacks were recorded. The prognosis was evaluated.

\section{Evaluation method}

Evaluation of epileptic symptoms. According to the classification criteria for seizures and symptoms of epilepsy established by ILAE, symptoms of epilepsy were divided into partial, spastic, typical absence, atypical absence, tonic-clonic, atonic, myoclonic and complex seizures. The numbers of patients experiencing each type in both groups were recorded.

Prognostic evaluation. Comprehensive evaluation was carried out according to the EEG before treatment and prognosis of patients after treatment. If the patient showed good indicators and good prognosis after treatment then the symptom was judged as false negative (FN); if indicators were good and prognosis was poor, true negative (TN); if
Table III. Comparison of post-treatment seizures (mean \pm SD).

\begin{tabular}{lcc}
\hline Groups & $\begin{array}{c}\text { No. of } \\
\text { seizures }(\mathrm{n})\end{array}$ & $\begin{array}{c}\text { Duration of each } \\
\text { seizure (min) }\end{array}$ \\
\hline Dynamic V-EEG & $7.7 \pm 3.64$ & $15 \pm 6.91$ \\
Conventional & $12.64 \pm 83$ & $21 \pm 7.48$ \\
P-value & $<0.01$ & $<0.05$
\end{tabular}

V-EEG, video-electroencephalogram.

both indicators and prognosis were poor, true positive (TP); if indicators were not good but prognosis was good after treatment, false positive (FP). The accuracy (accuracy = $(\mathrm{TP}+\mathrm{TN}) /$ number of patients $\mathrm{x} 100 \%)$, error rate (error rate $=$ $\mathrm{FP} /$ number of patients $\times 100 \%$ ) and specificity (specificity $=$ $\mathrm{TN} /(\mathrm{TN}+\mathrm{FP}) \times 100 \%)$ were calculated.

Statistical analysis. All the data were analyzed by SPSS 18.0 software (SPSS Inc., Chicago, IL, USA). The data were expressed as mean \pm standard deviation (SD). Student's t-test and variance analysis were used to compare the two independent samples. Countable data were tested using $\chi^{2}$ test or non-parametric rank sum test. A $\mathrm{P}<0.05$ was considered to indicate a statistically significant difference.

\section{Results}

General information and clinicopathological features of patients. Of the 200 patients, 109 were male and 91 were 
Table IV. Prognostic evaluation of epilepsy in the two groups.

\begin{tabular}{lcccccccc}
\hline Groups & $\mathrm{n}$ & $\mathrm{TN}(\mathrm{n})$ & $\mathrm{FN}(\mathrm{n})$ & $\mathrm{TP}(\mathrm{n})$ & $\mathrm{FP}(\mathrm{n})$ & Accuracy & Error rate & Specificity \\
\hline Dynamic V-EEG & 93 & 37 & 5 & 40 & 11 & $82.8 \%$ & $11.8 \%$ & $77.1 \%$ \\
Conventional & 76 & 25 & 13 & 21 & 18 & $60.5 \%$ & $23.7 \%$ & $58.1 \%$ \\
P-value & & & & & $<0.01$ & $<0.01$ & $<0.01$ \\
\hline
\end{tabular}

V-EEG, video-electroencephalogram.

female. The mean age was $8.28 \pm 3.56$ years. The course of disease and seizure frequency of the patients in both the dynamic V-EEG and conventional monitoring groups are shown in Table I. There were no significant differences in age, sex, course of disease or seizure frequency between the two groups $(\mathrm{P}>0.05)$. The different types of epilepsy symptoms are shown in Table II. There were no significant differences in epilepsy types between the two groups $(\mathrm{P}>0.05)$.

The detection of epileptic discharge. The epilepsy detection rate in the two groups is shown in Fig. 1. The detection rate in the dynamic V-EEG monitoring group was significantly higher than in the conventional monitoring group (93 vs. 76 cases, $\mathrm{P}<0.05)$.

The epilepsy detection rate at different time points. The rate of detection by dynamic V-EEG monitoring and conventional monitoring was analyzed and compared at different time points (Fig. 2). The detection rates by dynamic V-EEG monitoring at 1,2,3, and $4 \mathrm{~h}$ were $43,57,69$, and $76 \%$, respectively, all significantly higher than by conventional monitoring at the corresponding time point $(\mathrm{P}<0.01)$.

Comparison of post-treatment seizures. Patients with the same type of epilepsy in both groups were subjected to the same drug treatment. After 3 months of treatment, the frequency and duration of each seizure were recorded and analyzed (Table III). The results showed that the frequency and duration of each seizure in patients of dynamic V-EEG monitoring group were both significantly lower than those in patients of conventional monitoring group $(\mathrm{P}<0.01$ and $\mathrm{P}<0.05$, respectively).

Prognostic evaluation of epilepsy. Patients in both groups were rechecked 3 months after treatment and compared with the EEG data before treatment. The patients were divided into FN, TN, FP and TP, and the specificity, accuracy and error rate were calculated to evaluate prognosis (Table IV). The prognostic accuracy and specificity in the dynamic V-EEG were significantly higher than those in the conventional monitoring group, while the error rate in the dynamic V-EEG was significantly lower than in the conventional monitoring group $(\mathrm{P}<0.01)$.

\section{Discussion}

As a common form of epilepsy, pediatric epilepsy has recently attracted more attention in clinical practice (11). Due to the uncertainty of the time of onset and frequency of seizures, as

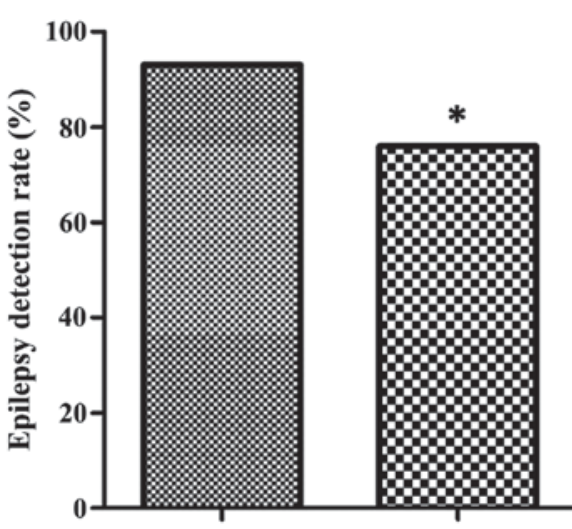

Dynamic V-EEG Conventional

Figure 1. The epilepsy detection rate in the two groups over $72 \mathrm{~h}$. The detection rate in the dynamic V-EEG monitoring group was significantly higher than in the conventional monitoring group $(" \mathrm{P}<0.05)$. V-EEG, videoelectroencephalogram.

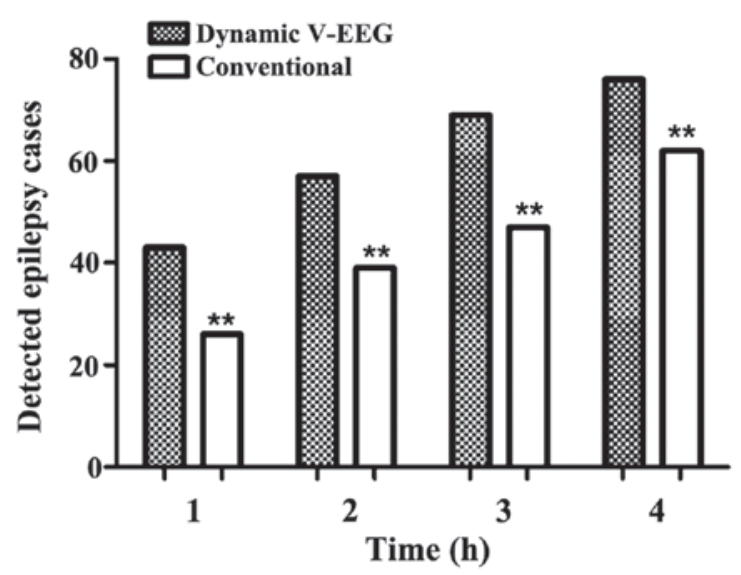

Figure 2. The epilepsy detection rate at different time points in each group. The detection rates by dynamic V-EEG monitoring at 1,2,3 and $4 \mathrm{~h}$ were all higher than those by conventional monitoring at the corresponding time point $\left({ }^{* *} \mathrm{P}<0.01\right)$. V-EEG, video-electroencephalogram.

well as inconsistent clinical manifestations, pediatric epilepsy has become a refractory disease (12-14). The pathogenesis of pediatric epilepsy is still unclear; previous studies have shown that pediatric epilepsy is related to abnormal brain development, genetic disease, brain infections, trauma and cerebrovascular diseases (15-17). At present, EEG is widely used in the detection of pediatric epilepsy. As a device to detect brain waves, EEG can effectively detect abnormal discharge of brain neurons and record whole brain waves at any time, 
including during exercise and sleep. Dynamic V-EEG can record both EEG and patients' clinical manifestations, which can then be combined for the diagnosis and classification of epilepsy (18).

Our study mainly discussed the application value of dynamic V-EEG in the diagnosis and prognostic evaluation of pediatric epilepsy. Conventional dynamic EEG monitoring was used as the control group. We found that dynamic EEG monitoring combined with video observation to monitor both EEG changes and the clinical manifestations of epilepsy can significantly increase the accuracy of the detection of epilepsy. In addition, dynamic V-EEG monitoring had the advantages of high accuracy, high specificity and low error rate. The most reliable basis for the diagnosis of epilepsy is EEG-clinical synchronous seizures and dynamic V-EEG can monitor both the EEG and clinical manifestations and use the combination of these to make a diagnosis. Dynamic V-EEG and conventional EEG were used to classify epilepsy types for appropriate treatment. We found that the prognosis was significantly better in the dynamic V-EEG than that in conventional monitoring group. In addition, the frequency and duration of each seizure in patients in the dynamic V-EEG were both significantly lower than those in patients in the conventional monitoring group. The results showed that dynamic V-EEG monitoring is more accurate for the diagnosis and classification of epilepsy than conventional dynamic EEG monitoring. As a digital EEG method derived from dynamic EEG, dynamic V-EEG not only can accurately localize the epileptiform discharges during playback analysis but also has great value in the classification of epilepsy $(19,20)$.

In conclusion, dynamic V-EEG has several advantages over conventional EEG. Dynamic V-EEG can significantly increase the accuracy of diagnosis, so as to ensure appropriate symptomatic treatment. Therefore, dynamic V-EEG has great clinical value in the diagnosis and prognosis of epilepsy and will likely become one of the most commonly used and most reliable tools in the treatment of epilepsy.

\section{References}

1. Peljto AL, Barker-Cummings C, Vasoli VM, Leibson CL, Hauser WA, Buchhalter JR and Ottman R: Familial risk of epilepsy: A population-based study. Brain 137: 795-805, 2014.

2. Brodie MJ, Besag F, Ettinger AB, Mula M, Gobbi G, Comai S, Aldenkamp AP and Steinhoff BJ: Epilepsy, antiepileptic drugs and aggression: an evidence-based review. Pharmacol Rev 68: 563-602, 2016

3. Kobau R, Cui W, Kadima N, Zack MM, Sajatovic M, Kaiboriboon $\mathrm{K}$ and Jobst B: Tracking psychosocial health in adults with epilepsy - estimates from the 2010 National Health Interview Survey. Epilepsy Behav 41: 66-73, 2014
4. Cui W, Zack MM, Kobau R and Helmers SL: Health behaviors among people with epilepsy - results from the 2010 National Health Interview Survey. Epilepsy Behav 44: 121-126, 2015.

5. Yang RR, Wang WZ, Snape D, Chen G, Zhang L, Wu JZ, Baker GA, Zheng XY and Jacoby A: Stigma of people with epilepsy in China: Views of health professionals, teachers, employers and community leaders. Epilepsy Behav 21: 261-266, 2011.

6. Kaiboriboon K, Bakaki PM, Lhatoo SD and Koroukian S: Incidence and prevalence of treated epilepsy among poor health and low-income Americans. Neurology 80: 1942-1949, 2013.

7. Lamberts RJ, Blom MT, Wassenaar M, Bardai A, Leijten FS, de Haan GJ, Sander JW, Thijs RD and Tan HL: Sudden cardiac arrest in people with epilepsy in the community: Circumstances and risk factors. Neurology 85: 212-218, 2015.

8. Berg AT, Baca CB, Loddenkemper T, Vickrey BG and Dlugos D: Priorities in pediatric epilepsy research: Improving children's futures today. Neurology 81: 1166-1175, 2013.

9. Poduri A and Lowenstein D: Epilepsy genetics - past, present and future. Curr Opin Genet Dev 21: 325-332, 2011.

10. Thompson AW, Kobau R, Park R and Grant D: Epilepsy care and mental health care for people with epilepsy: California health interview survey, 2005. Prev Chronic Dis 9: E60, 2012.

11. Bowen JM, Snead OC, Chandra K, Blackhouse G and Goeree R: Epilepsy care in ontario: An economic analysis of increasing access to epilepsy surgery. Ont Health Technol Assess Ser 12: $1-41,2012$.

12. Hesdorffer DC, Beck V, Begley CE, Bishop ML, CushnerWeinstein S, Holmes GL, Shafer PO, Sirven JI and Austin JK: Research implications of the Institute of Medicine Report, epilepsy across the spectrum: Promoting Health and Understanding. Epilepsia 54: 207-216, 2013.

13. Berg AT and Plioplys S: Epilepsy and autism: Is there a special relationship? Epilepsy Behav 23: 193-198, 2012.

14. Berg AT, Loddenkemper T and Baca CB: Diagnostic delays in children with early onset epilepsy: Impact, reasons and opportunities to improve care. Epilepsia 55: 123-132, 2014.

15. Burneo JG, Jette N, Theodore W. Disparities in epilepsy: Report of a systematic review by the North American Commission of the International League Against Epilepsy. Epilepsia 50: 42-52, 2009.

16. Wirrell EC, Grossardt BR, Wong-Kisiel LC-L and Nickels KC: Incidence and classification of new-onset epilepsy and epilepsy syndromes in children in Olmsted County, Minnesota from 1980 to 2004: A population-based study. Epilepsy Res 95: 110-118, 2011.

17. Sidhu MK, Stretton J, Winston GP, Symms M, Thompson PJ, Koepp MJ and Duncan JS: Factors affecting reorganisation of memory encoding networks in temporal lobe epilepsy. Epilepsy Res 110: 1-9, 2015.

18. Burneo JG, Shariff SZ, Liu K, Leonard S, Saposnik G and Garg AX: Disparities in surgery among patients with intractable epilepsy in a universal health system. Neurology 86: 72-78, 2016.

19. Walker NM, Jackson DC, Dabbs K, Jones JE, Hsu DA, Stafstrom CE, Sheth RD, Koehn MA, Seidenberg M and Hermann BP: Is lower IQ in children with epilepsy due to lower parental IQ? A controlled comparison study. Dev Med Child Neurol 55: 278-282, 2013.

20. Mbuba CK, Ngugi AK, Fegan G, Ibinda F, Muchohi SN, Nyundo C, Odhiambo R, Edwards T, Odermatt P, Carter JA, et al: Risk factors associated with the epilepsy treatment gap in Kilifi, Kenya: A cross-sectional study. Lancet Neurol 11: 688-696, 2012. 